\title{
Selective effects of temperature on some enzyme polymorphisms in laboratory populations of Drosophila melanogaster
}

\author{
J. G. Oakeshott*, \\ S. R. Wilson $\dagger$ \\ and P. Parnell* ${ }^{*}$
}

\author{
* Department of Population Biology, Research School \\ of Biological Sciences, The Australian National \\ University, P.O. Box 475, Canberra City, A.C.T. 2601, \\ Australia. \\ $\dagger$ Department of Statistics, Research School of Social \\ Sciences, The Australian National University, \\ P.O. Box 4, Canberra, A.C.T. 2600, Australia. \\ $\ddagger$ Department of Animal Science, University of New \\ England, Armidale, N.S.W. 2351, Australia.
}

In the first experiment reported here 44 isofemale lines from four recently collected base populations were each divided into three selection lines, one for each of three temperature environments-constant $23^{\circ} \mathrm{C}$, constant $29^{\circ} \mathrm{C}$ and diurnally varying $17^{\circ} \mathrm{C} / 29^{\circ} \mathrm{C}$. Allele frequency responses after 30 generations of selection were similar across the three environments for the $A d h, G p d h$ and $P g m$ polymorphisms but differed among the four base populations for $A d h$ and $G p d h$. Equilibrium $A d h^{F}$ and $G p d h^{S}$ frequencies predicted from maximum likelihood estimates of selection coefficients were higher for base populations from more temperate latitudes; this was consistent with latitudinal clines previously reported in $A d h^{F}$ and $G p d h^{S}$ frequencies. By contrast, the equilibrium frequencies predicted for $P g m^{1.00}$ were generally high for all base populations; however this was also consistent with geographic data on $P_{g m}{ }^{1.00}$ frequencies, which are uniformly high over a wide range of latitudes.

In a second experiment involving greater temperature stress, adults from eight recent collections were exposed to $38^{\circ} \mathrm{C}$ for an hour. Differences in survival percentages were then evident among $G$ pdh, but not $A d h$, genotypes. (Pgm genotypes were not consistently scorable after the heat shock.) Survival percentages were highest for $G p d h^{F} / G p d h^{F}$; intermediate for $G p d h^{F} / G p d h^{S}$ and lowest for $G p d h^{S} / G p d h^{S}$.

From the first experiment it was suggested that the latitudinal clines for $A d h$ and $G p d h$ in part reflect latitudinal variation in the selection imposed by the internal environments of the flies, as determined by their genetic backgrounds. The second experiment suggested that the $G p d h$ clines also reflect direct selective effects of stressfully high temperatures.

\section{INTRODUCTION}

It has been an elusive goal of population genetics over the last two decades, not only to test for the action of natural selection on enzyme polymorphisms, but also to elucidate the underlying mechanisms of the selection. Unfortunately, very few conclusive results addressing either of these issues have been obtained. However, large-scale latitudinal associations of allele frequencies have recently been found to recur across continents and hemispheres for six of ten enzyme polymorphisms studied in Drosophila melanogaster; it was concluded that these associations could only reasonably be explained by natural selection (Oakeshott et al., $1984 b$ for a summary). The present paper describes experiments designed to test the possibility that the mechanism of selection operating on two of the latitudinally varying polymorphisms is associated with variation in ambient temperature.

The two polymorphic loci are alcohol dehydrogenase $(A d h)$ and glycerol-3-phosphate dehydrogenase $(G p d h)$. These were chosen because differences in the thermal properties of the purified 
allozymes (Zera et al., 1983 for a review) are in both cases in the right direction to explain not only their latitudinal clines but also the seasonal variation of their allele frequencies reported in some populations (Berger, 1971; Miller et al., 1975; Gionfriddo and Vigue, 1978; Franklin, 1981; Cavener and Clegg, 1981). Yet no consistent results have emerged to date from laboratory studies testing for corresponding fitness differences among $A d h$ and $G p d h$ genotypes at different temperatures. From the biochemical data it would be predicted that the $A d h^{S}$ and $G p d h^{F}$ alleles would be selectively advantageous at high temperatures. For $A d h$, significant differences in allele or genotype frequencies consistent with this have been found by Johnson and Powell (1974), van Delden et al. (1978), van Delden and Kamping (1980) and Sampsell and Sims (1982), but not by Milkman (1977), Oakeshott (1979), McKechnie et al. (1981) and Vigue et al. (1982). For Gpdh, fitness differences predicted by the biochemical data have been reported by McKechnie et al. (1981) and Barnes and Laurie-Ahlberg (1985) but not by Milkman (1977), Oakeshott (1979) or Schenfeld and McKechnie (1979).

Oakeshott et al. (1984a) have pointed to similar discrepancies among laboratory studies on the selective effects of ethanol on the $A d h$ polymorphism in $D$. melanogaster. A relationship between ethanol in the media and $A d h$ allele or genotype frequencies can generally only be found if the doses of ethanol used are sufficiently high to cause heavy mortality and if the study populations have spent a period of years in the laboratory prior to the experiment.

Here we examine whether analogous effects might explain the discrepancies among the studies screening for selection on $A d h$ and Gpdh due to different temperature regimes. The study populations are derived from recent field collections and two types of temperature regime are investigated: continuous exposures to different relatively mild temperatures; and short term exposures to relatively high temperatures causing heavy mortality. In addition to $A d h$ and $G p d h$, the phosphoglucomutase $(\mathrm{Pgm})$ polymorphism is also studied. Unlike the first two systems, Pgm allele frequencies are not clinally related to latitude in any continent so far studied (Oakeshott et al., 1981).

\section{MATERIALS AND METHODS}

\section{Continuous exposures to mild temperatures}

Since only small differences might be expected in the selective effects of different relatively mild tem- peratures, these conditions were applied continuously for 30 generations and the selection coefficients were estimated from allele frequency changes observed over this interval.

About 30 isofemale lines were established from base populations collected at each of four localities from a range of latitudes along the eastern seaboard of Australia. The localities were Redbanks $\left(27 \cdot 5^{\circ} \mathrm{S}, 153.0^{\circ} \mathrm{E}\right)$, Craigmoor $\left(32.6^{\circ} \mathrm{S}, 149 \cdot 6^{\circ} \mathrm{E}\right)$, Rothbury $\left(32 \cdot 8^{\circ} \mathrm{S}, 151 \cdot 3^{\circ} \mathrm{E}\right)$ and Cygnet $\left(43 \cdot 0^{\circ} \mathrm{S}\right.$, $147 \cdot 3^{\circ} \mathrm{E}$ ). Twenty flies from the first generation of each isofemale line were scored for $A d h, G p d h$ and $P g m$ phenotypes (electrophoretic methods in Oakeshott et al., 1981; 1982) and between 10 and 12 of the lines from each base population which were segregating for $A d h, G p d h$ and, where possible, Pgm were chosen for further use. Each of the 44 lines chosen was amplified for two generations and then divided into four cohorts. One cohort of up to about 100 flies was used to score $A d h, G p d h$ and $P g m$ allele frequencies again. The other three cohorts, each of at least 150 flies, were used to establish three selection lines thereafter to be kept under different temperature environments: constant $23^{\circ} \mathrm{C}$, constant $29^{\circ} \mathrm{C}$ and diurnally ( 12 hour $/ 12$ hour) varying $17^{\circ} \mathrm{C} / 29^{\circ} \mathrm{C}$. All selection lines were maintained by mass transfer in $250 \mathrm{ml}$ bottles for 30 discrete generations. The feed medium for all three environments was the same and is described in Oakeshott and Gibson (1981).

Five $23^{\circ}$ lines, two $29^{\circ}$ lines and five $17^{\circ} / 29^{\circ}$ lines were discarded during the course of the experiment when the number of aduits available in each line for founding a new generation fell below about 50. Excluding the lines discarded, the generation time (days) and number of adults transferred each generation $(\bar{x} \pm$ S.E. $)$ were 23 and $235 \pm$ 17 for $23^{\circ}$ lines, 15 and $163 \pm 15$ for $29^{\circ}$ lines and 28 and $128 \pm 15$ for $17^{\circ} / 29^{\circ}$ lines.

At generations 10,20 and 30 all selection lines were rescored for $A d h, G p d h$ and, where segregating, $P g m$ allele frequencies. The average numbers $(\bar{x} \pm$ S.E.) of flies from each selection line scored for generations $0,10,20$ and 30 respectively were: $85 \pm 3,62 \pm 1,66 \pm 2$ and $65 \pm 2$ for $A d h ; 79 \pm 4$, $63 \pm 2,65 \pm 3$ and $62 \pm 3$ for $G p d h$; and $75 \pm 6,84 \pm$ 3, $91 \pm 4$ and $99 \pm 4$ for $P g m$. These numbers were consistent across the three temperature environments.

The conventions for nomenclature and the statistical methods for estimating the selection coefficients $s$ and $t$ were as described in Wilson $e t$ al. (1982) and Wilson and Oakeshott (1985). Each selection line was denoted by a three symbol code $(b, l, e)$ defining it in terms of three factors: the base population from which it originated $(b=$ 
Redbanks, Craigmoor, Rothbury and Cygnet), the isofemale line from which it was derived $(l=$ $1 \ldots 11$ for Redbanks, $1 \ldots 12$ for Craigmoor, $1 \ldots 10$ for Rothbury and $1 \ldots 11$ for Cygnet) and the temperature environment to which it was allocated $\left(e=23^{\circ}, 29^{\circ}, 17^{\circ} / 29^{\circ}\right)$. Selection coefficients were estimated by maximum likelihood methods for each selection line and for various groups of them defined by $b, l$ or $e$ values. Differences among the coefficients for the different selection lines and their groups were assessed by analysis of deviance.

\section{Short term exposures to high temperatures}

It was expected that the high temperature shocks would impose stronger selective pressures than the milder temperatures tested above. Therefore the high temperature shocks were administered within a single generation and selection differentials were calculated from differences in the genotypic numbers among the survivors and casualties of the shocks. Because of stability problems with the enzyme, $P g m$ genotypes were difficult to score after the heat shocks and were not included in the analysis.

At least 40 adults were collected from each of eight Australian localities; Ipswich and Redbanks (about $20 \mathrm{~km}$ apart at $27 \cdot 5^{\circ} \mathrm{S}, 153 \cdot 0^{\circ} \mathrm{E}$ ), Bellevue, Elliott, Lindeman, Rothbury and Tulloch (all between $1 \mathrm{~km}$ and $10 \mathrm{~km}$ apart at $32 \cdot 8^{\circ} \mathrm{S}, 151 \cdot 3^{\circ} \mathrm{E}$ ) and Cygnet $\left(43 \cdot 0^{\circ} \mathrm{S}, 147 \cdot 3^{\circ} \mathrm{E}\right)$. Populations founded from these collections were tested for heat tolerance within three laboratory generations of their capture. For each population the tests were carried out on about seven replicate cohorts, each of 100-150 adults aged 6-8 days at $25^{\circ} \mathrm{C}$ since emergence. Tolerance was tested by placing each cohort in a stoppered vial submerged in a $38^{\circ} \mathrm{C}$ water bath for an hour. Three hours after removing the vials from the bath the dead and living flies were separated and scored for Adh. For Cygnet, both $A d h$ and $G p d h$ were scored.

\section{RESULTS}

\section{Continuous exposures to mild temperatures}

All 44 isofemale lines used to establish the selection lines were initially polymorphic for $A d h^{F}$ and $A d h^{S}$ and $G p d h^{F}$ and $G p d h^{S}$. Initially 18 of the isofemale lines were also polymorphic for Pgm variants, eight for $\mathrm{Pgm}^{1.00}$ and $\mathrm{Pgm}^{1.20}$ and 10 for $\mathrm{Pgm}^{1.00}$ and $\mathrm{Pgm}^{0.90}$. (The 26 other isofemale lines were monomorphic for $\mathrm{Pgm}^{1.00}$.) The 44 lines had been chosen to represent a wide range of initial frequencies of the $A d h$ and $G p d h$ variants within each of the four base populations. However, in all 18 isofemale lines polymorphic for $\mathrm{Pgm}$ the $\mathrm{Pgm}^{1 \cdot 00}$ allele was initially at a frequency above 50 per cent.

The changes in $A d h^{F}$ frequencies over time are shown graphically in fig. 1 for each selection line. Any general trends in $A d h^{F}$ frequency change across temperature environments, base populations or initial $A d h^{F}$ frequencies are largely obscured by variation among selection lines within each of these categories and by reversals in the directions of change over time within lines. However, some general patterns emerge if net changes in $A d h^{F}$ frequency over the course of the experiment are computed (table 1). Significantly more selection lines showed a net rise in $A d h^{F}$ frequency than showed a net fall. This trend is consistent throughout the range of initial $A d h^{F}$ frequencies and across the three temperature environments but not across the four base populations. A majority of Craigmoor, Rothbury and, in particular, Cygnet selection lines showed a net rise $\left(\chi_{2}^{2}\right.$ for homogeneity $=2 \cdot 5, P>0.05 ; \chi_{1}^{2}$ for $1: 1=$ $12 \cdot 6, P<0 \cdot 001)$ but arnong Redbanks lines, more, albeit not significantly more $\left(\chi_{1}^{2}=0 \cdot 8, P>0 \cdot 05\right)$, showed a net fall.

There was a trend for correlated net frequency changes across the three selection lines derived from each isofemale line. Thus, in each of 17 of the 41 isofemale lines for which $A d h$ data were available from all three environments, all three selection lines showed the same direction of net $A d h^{F}$ frequency change. This is significantly more than expected by chance $\left(\chi_{1}^{2}=5.9, P<0.05\right)$.

Changes in $G p d h^{F}$ frequencies over time are shown graphically in fig. 2 but once again any general trends are largely obscured by variation within and between individual lines. Computation of net frequency changes in this case reveals no overall difference between the numbers of lines showing rises and falls (table 1). This similarity applied throughout the range of initial $G p d h^{F}$ frequencies and across the three temperature environments. However, as was the case for $A d h^{F}$, the base populations differed significantly in the ratio of net rises and falls in $G p d h^{F}$ frequency. A consistent $\left(\chi_{2}^{2}=3.9, \quad P>0.05\right)$ and significant $\left(\chi_{1}^{2}=7 \cdot 2, P<0 \cdot 01\right)$ excess of net rises occurred among Redbanks, Craigmoor and Rothbury lines but among Cygnet lines there was a nearly significant excess of falls $\left(\chi_{1}^{2}=3 \cdot 7, P=0 \cdot 06\right)$. And, again like $A d h$, there was a significant excess $\left(\chi_{1}^{2}=\right.$ $8 \cdot 5, P<0.01)$ of isofemale lines (18 in 40$)$ in each of which the direction of change was the same for all three selection lines. 


\section{Temperature Environments}

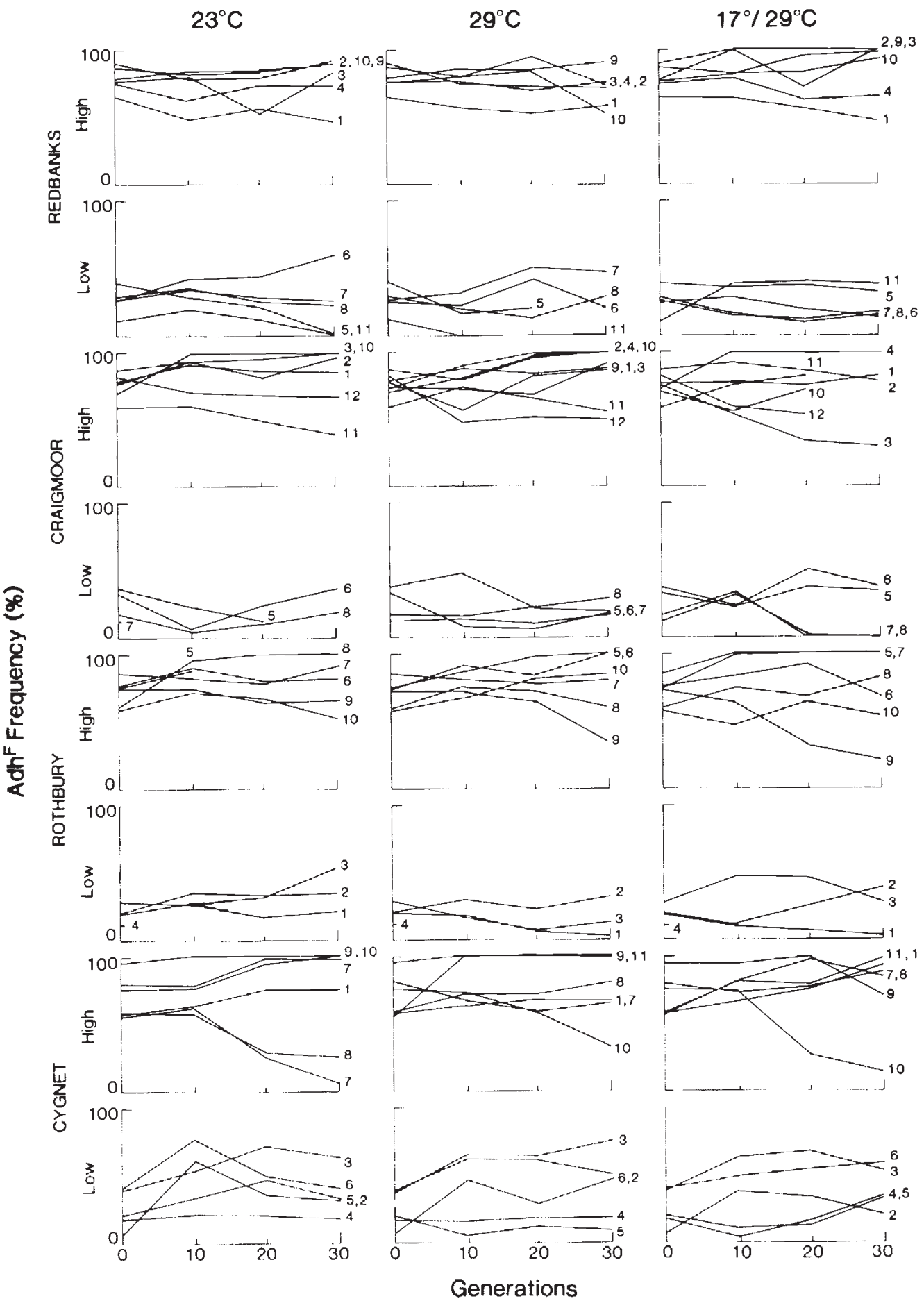

Figure 1 Changes in $A d h^{+}$frequency over the 30 generations of selection. Each graph shows the results for all selection lines in a particular base population-temperature environment-initial frequency class (high $=A d h^{\Gamma}>50$ per cent; low $=A d h^{F}<50$ per cent) combination. Numbers identifying the isofemale lines within each base population are given on the right-hand side of each graph. 
Table 1 The numbers of selection lines showing net rises and falls (rises: falls) in allele frequencies from generation 0 to generation 30 (or if discarded earlier, to the last generation scored). Values of $\chi_{1}^{2}$ are shown which test the total data for agreement with equal numbers of rises and falls. Values of $\chi^{2}$ are also given which test the homogeneity of the ratios of rises to falls among the two initial frequency classes, the three temperature environments and the four base populations from which the selection lines were derived.

\begin{tabular}{|c|c|c|c|c|c|}
\hline & \multirow[b]{2}{*}{$A d h^{F}$} & \multirow[b]{2}{*}{$G p d h^{F}$} & \multicolumn{3}{|c|}{$P g m^{1 \cdot 00}$} \\
\hline & & & with $\mathrm{Pgm}^{1 \cdot 20}$ & with $P g m^{0.90}$ & Combined \\
\hline Overall & $77: 48$ & $71: 56$ & $18: 6$ & $19: 11$ & $37: 17$ \\
\hline$\chi_{1}^{2}(1: 1)$ & $6 \cdot 7 \dagger$ & 1.8 & $6 \cdot 0^{*}$ & $2 \cdot 1$ & $7 \cdot 4 \dagger$ \\
\hline \multicolumn{6}{|l|}{ Initial frequency } \\
\hline : high & $47: 28$ & $33: 33$ & & & \\
\hline : low & $30: 20$ & $38: 23$ & & & \\
\hline$\chi_{1}^{2}$ (homog.) & $0 \cdot 1$ & 1.5 & & & \\
\hline \multicolumn{6}{|l|}{ Temperature } \\
\hline$: 22^{\circ}$ & $28: 12$ & $24: 17$ & $4: 4$ & $7: 3$ & $11: 7$ \\
\hline$: 29^{\circ}$ & $24: 19$ & $22: 22$ & $7: 1$ & $7: 3$ & $14: 4$ \\
\hline$: 17^{\circ} / 29^{\circ}$ & $25: 17$ & $25: 17$ & $7: 1$ & $5: 5$ & $12: 6$ \\
\hline$\chi_{2}^{2}$ (homog.) & 1.9 & $4 \cdot 1$ & $4 \cdot 0$ & $1 \cdot 2$ & $1 \cdot 2$ \\
\hline \multicolumn{6}{|l|}{ Populations } \\
\hline Redbanks & $14: 19$ & $17: 16$ & $9: 3$ & $3: 0$ & $12: 3$ \\
\hline : Craigmoor & $20: 12$ & $24: 8$ & - & $11: 1$ & $11: 1$ \\
\hline Rothbury & $17: 10$ & $19: 10$ & $5: 1$ & $3: 3$ & $8: 4$ \\
\hline Cygnet & $26: 7$ & $11: 22$ & $4: 2$ & $2: 7$ & $6: 9$ \\
\hline$\chi_{3}^{2}$ (homog.) & $9 \cdot 3^{*}$ & $12.9+$ & 0.5 & $16 \cdot 1 \dagger$ & $9 \cdot 6^{*}$ \\
\hline
\end{tabular}

$* P<0.05 . \quad+P<0.01$.

The results for both $P g m$ polymorphisms are shown graphically in fig. 3 and summarised in terms of net $\mathrm{Pgm}^{1 \cdot 00}$ frequency changes in table 1 . A significant majority of lines polymorphic for $\mathrm{Pgm}^{1 \cdot 00}$ and $\mathrm{Pgm}^{1 \cdot 20}$ showed a net rise in $\mathrm{Pgm}^{1 \cdot 00}$ frequency and this trend was consistent across temperature environments and base populations. Among selection lines polymorphic for $\mathrm{Pgm}^{1 \cdot 00}$ and $P \mathrm{gm}^{0.90}$ there was no overall difference in the numbers showing net rises and falls in $\operatorname{Pgm}^{1.00}$ frequencies. However, this overall similarity concealed significant heterogeneity among the four base populations; selection lines from Redbanks, Craigmoor and Rothbury were consistent $\left(\chi_{2}^{2}=\right.$ $5.3, P>0.05)$ in showing a significant excess of rises $\left(\chi_{1}^{2}=8 \cdot 1, P<0 \cdot 01\right)$, whereas Cygnet lines showed an excess of falls. Once again there was a significant excess of isofemale lines ( 10 out of 18 , $\chi_{1}^{2}=9.0, P<0.01$ for the two $P g m$ polymorphisms combined) in each of which the direction of net $P g m^{1 \cdot 00}$ frequency change was the same in all three selection lines.

Comparison of net frequency changes thus suggested that some systematic changes in allele frequencies occurred for Adh, Gpdh and Pgm and that these changes, while largely independent of initial allele frequencies and temperature environments, differed among base populations and isofemale lines within base populations. The next step is to estimate the selection coefficients $s$ and $t$ and to test them for significant differences, first from zero, and then due to factors such as different base populations $(b)$, isofemale lines $(l)$ and temperature environments $(e)$. The coefficients are estimated by maximum likelihood and tested for significance by analyses of deviance. These statistical procedures are described in full by Wilson and Oakeshott (1985) and their applications to other data sets from analogous experiments are described by Wilson et al. (1982), Oakeshott et al. (1983) and Oakeshott et al. (1984a). The selection coefficients estimated indicate net differences in fitness: no assumptions are made about the contributions to the differences made by the various components of fitness.

The significance tests are based on deviance values calculated for different models of selection on each polymorphism. The deviance is simply a measure of the goodness of fit of a model to the observed. The difference in deviance is then calculated between models differing only by a single factor (e.g., temperature environments). This difference in deviance is distributed as a $\chi^{2}$, so that the significance of effects due to that factor is assessed simply by reference to a standard $\chi^{2}$ table. (Degrees of freedom are given by the difference in the numbers of parameters (i.e. $s$ and $t$ values) fitted in the two models.)

The present experimental design permits seven models of varying complexity to be fitted for each polymorphism. The first model is the simplest, a neutral $(0,0,0)$ model with zero $s$ and $t$ in all 


\section{Temperature Environments}
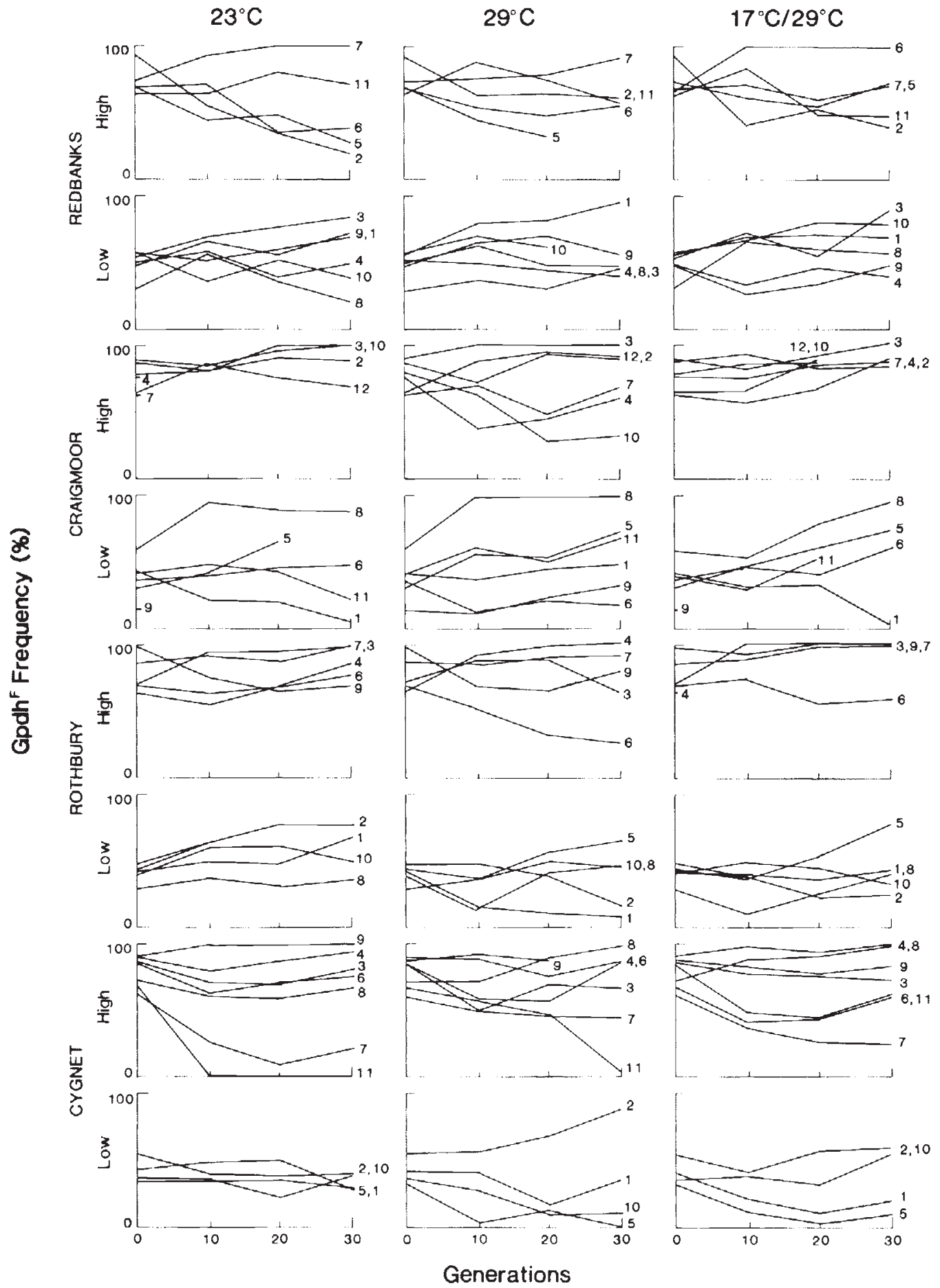

Figure 2 Changes in $G p d h^{F}$ frequency over the 30 generations of selection. The format is the same as Figure 1 except that the threshold dividing "high" and "low" initial Gpdhr frequencies is set at 60 per cent. (Initial frequencies were higher overall for $G p d h^{F}$ than $A d h^{F}$.) 


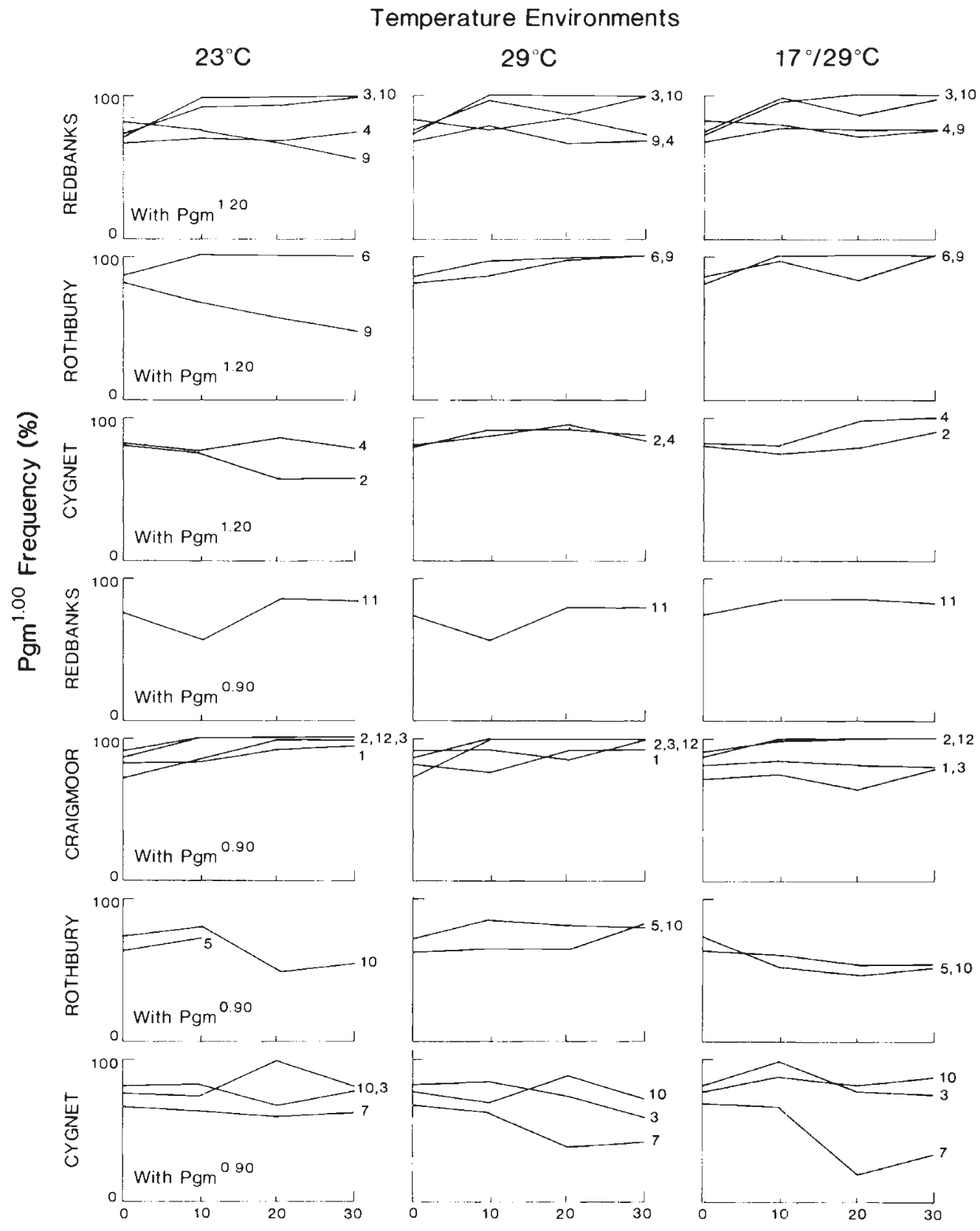

Generations

Figure 3 Changes in $\operatorname{Pgm}^{1 \cdot 00}$ frequencies over the 30 generations of selection. Results for the $P g m^{1 \cdot 20}-P m^{1 \cdot 00}$ polymorphism are shown separately from those for the $P_{g m^{0.90}}-P_{g m^{1 \cdot 00}}$ polymorphism.

selection lines. The difference in deviance from the first model provides a test for the second model $(., .,$.$) , which assumes that common non-zero s$ and $t$ values apply in all lines. The third and fourth models are both tested by their deviance differences from the second; the third $(., ., e)$ allows $s$ and $t$ to vary among the three temperature environments and the fourth $(b, \ldots$,$) allows s$ and $t$ to 
vary among the four base populations. The fifth and sixth models are tested against the fourth, the fifth $(b, ., e)$ varying $s$ and $t$ among temperature environments within base populations and the sixth $(b, l,$.$) varying s$ and $t$ among isofemale lines within base populations. The seventh model $(b, l, e)$, which is tested against the sixth, allows $s$ and $t$ to vary among temperature environments within isofemale lines (i.e., it fits a different $s$ and $t$ value for every selection line).

The magnitudes of the deviance values and their differences increase with the effective population size assumed in the model fitting (because the contribution which random effects can make towards an explanation of allele frequency change decreases). In this experiment we have data on observed population sizes (see MATERIALS AND METHODS) and the ratio, $f$, of effective to observed population sizes can be estimated by Monte Carlo simulations carried out as in Wilson and Oakeshott (1985). Results from these simulations were consistent across determinations for the different polymorphisms and suggested that the 95 per cent confidence limits for $f$ were approximately 0.2 and $0 \cdot 5$. This agrees well with the ranges calculated by the same simulation method in our previous experiments with bottle cultures (Oakeshott et al., 1984a; Wilson and Oakeshott, 1985).

For $A d h$, table 2 shows that no model gave a deviance decrease which was statistically significant at both $f=0 \cdot 2$ and $f=0 \cdot 5$. However, differences in the selection imposed in different isofemale lines within base populations $(b, l,$. were highly significant at $f=0.5$ and closely approached significance at $f=0 \cdot 2$. It is also noteworthy that selection regimes appeared to differ among base populations $(b, .,$.$) ,$ notwithstanding that these differences were only significant at $f=0.5$; an earlier experiment (Oakeshott et al., 1984a) had revealed systematic differences in selection regimes among base populations according to their latitude and this trend is reexamined below for the present data.

For $G p d h$, there were highly significant differences in selection regimes among base populations $(b, .,$.$) and isofemale lines within base popula-$ tions $(b, l,$.$) , at both f=0.2$ and 0.5 (table 2).

For the $P g m^{1 \cdot 20}-P g m^{1.00}$ polymorphism, the only model with significant effects at both $f$ values was that invoking the same non-zero $s$ and $t$ coefficients in all selection lines $(., .,$.$) . For$ $\mathrm{Pgm}^{0.90}-\mathrm{Pgm}^{1 \cdot 00}$, the clearest indications of selection were obtained from the differences in selective effects among base populations $(b, . .$.$) , which$ were highly significant at $f=0.5$ and approached significance at $f=0.2$ (table 2).

One clear result for all four polymorphisms at both $f$ values was thus the absence of significant selective effects of temperature consistent across base populations $(., ., e)$. There was some equivocal evidence of temperature effects in particular base populations $(b, ., e)$ or lines within base populations $(b, l, e)$, but these were never significant at both $f$ values.

On the other hand, selective-effects of base population consistent across temperatures were indicated for $A d h, G p d h$ and $P g m^{0.90}-P g m^{1 \cdot 00}$. For $P g m^{1 \cdot 20}-P g m^{1.00}$ selective effects consistent both across populations and temperatures were revealed. Therefore, fig. 4 gives the 95 per cent and 99 per cent confidence contours for the selection coefficients $s$ and $t$ calculated over all selection lines for $P g m^{1 \cdot 20}-P g m^{1 \cdot 00}$ and over all lines within each base population for $A d h, G p d h$ and $P g m^{0.90}$

Table 2 Deviance differences $(\Delta D)$ for testing the improvements in fit of a series of successively more parameterised models of selection to explain the observed allele frequency changes. $f$ is the ratio of effective to actual population size assumed during model fitting. $(., \ldots)=$ common, non-zero $s$ and $t$ in all selection lines; $(., \ldots)=s$ and $t$ vary across the three temperature environments; $(b, .,)=$.$s and t$ vary across the four base populations; $(b, \ldots)=s$ and $t$ vary across temperatures within base populations; $(b, l,)=$.$s and t$ vary across isofemale lines within base populations; $(b, l, e)=s$ and $t$ vary across temperatures within isofemale lines.

\begin{tabular}{|c|c|c|c|c|c|c|c|c|c|c|c|c|}
\hline \multirow[b]{2}{*}{$\begin{array}{l}\text { Model } \\
\text { tested }\end{array}$} & \multicolumn{3}{|c|}{$A d h$} & \multicolumn{3}{|c|}{$G p d h$} & \multicolumn{3}{|c|}{$P g m^{1 \cdot 20}-P g m^{1 \cdot 00}$} & \multicolumn{3}{|c|}{$P g m^{0.90}-P g m^{1.00}$} \\
\hline & d.f. & $\begin{array}{l}\Delta D \mathrm{at} \\
f=0 \cdot 2\end{array}$ & $\begin{array}{l}\Delta D \text { at } \\
f=0.5\end{array}$ & d.f. & $\begin{array}{l}\Delta D \text { at } \\
f=0.2\end{array}$ & $\begin{array}{l}\Delta D \text { at } \\
f=0.5\end{array}$ & d.f. & $\begin{array}{l}\Delta D \text { at } \\
f=0 \cdot 2\end{array}$ & $\begin{array}{l}\Delta D \text { at } \\
f=0.5\end{array}$ & d.f. & $\begin{array}{l}\Delta D \text { at } \\
f=0 \cdot 2\end{array}$ & $\begin{array}{l}\Delta D \text { at } \\
f=0.5\end{array}$ \\
\hline$(., .,)$. & 2 & $2 \cdot 8$ & $7 \cdot 3^{*}$ & 2 & 0.8 & 1.5 & 2 & $6.6^{*}$ & $15 \cdot 0 \ddagger$ & 2 & $2 \cdot 2$ & $6 \cdot 5^{*}$ \\
\hline$(., ., e)$ & 4 & $0 \cdot 2$ & $0 \cdot 3$ & 4 & $1 \cdot 1$ & 1.7 & 4 & $2 \cdot 4$ & $5 \cdot 5$ & 4 & $1 \cdot 1$ & $2 \cdot 6$ \\
\hline$(b, .,)$. & 6 & $7 \cdot 9$ & $12 \cdot 6^{*}$ & 6 & $26 \cdot 4 \ddagger$ & $62 \cdot 3 \ddagger$ & 4 & $3 \cdot 4$ & $8.0 \S$ & 6 & $11 \cdot 3 \S$ & $25 \cdot 5 \ddagger$ \\
\hline$(b, l, e)$ & 152 & $129 \cdot 3$ & $239 \cdot 8 \ddagger$ & 150 & $124 \cdot 3$ & $252 \cdot 1 \ddagger$ & 32 & $22 \cdot 4$ & $41 \cdot 1$ & 40 & $21 \cdot 3$ & $35 \cdot 3$ \\
\hline
\end{tabular}

$\S P<0 \cdot 10$.

* $P<0.05$.

$\dagger P<0.01$.

$\ddagger P<0.001$. 

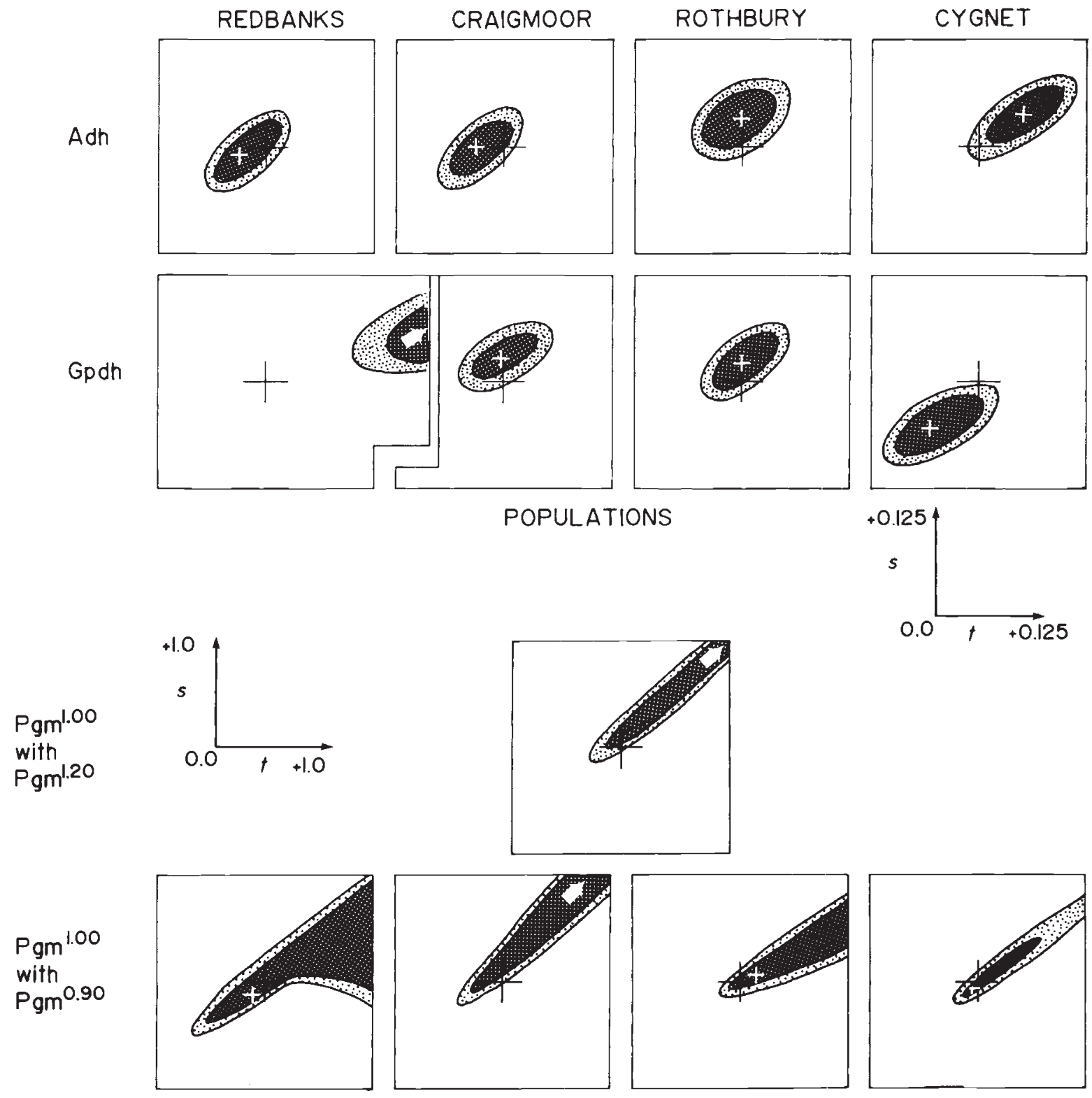

Figure 4 The 95 per cent and 99 per cent confidence contours for the maximum likelihood estimates of $s$ and $t$ in each base population for $A d h, G p d h$ and $P g m^{0.90}-P m^{1 \cdot 00}$ and across all base populations for $P g m^{1 \cdot 20}-P g m^{1 \cdot 00}$. Values of $s$ apply to the fitnesses of $A d h^{F}, G p d h^{F}$ and $P g m^{1 \cdot 00}$ homozygotes relative to their opposite homozygotes and are given vertically. Values of $t$ for the fitnesses of the heterozygotes, again relative to these opposite homozygotes, are given horizontally. The best estimates of $s$ and $t$ are indicated by white crosses or, where off-scale, by arrows. Areas inside 95 per cent confidence contours are most heavily stippled and areas outside 99 per cent contours are unshaded. Origins $(s=t=0)$ are given by black crosses.

$\mathrm{Pgm}^{1 \cdot 00}$. The coefficients defined relative fitnesses of

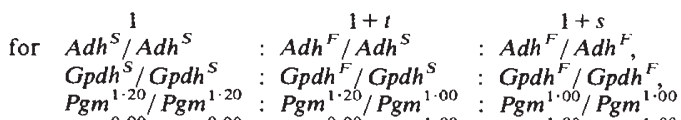

$$
\begin{aligned}
& \text { and } \mathrm{Pgm}^{0.90} / \mathrm{Pgm}^{0.90}: \mathrm{Pgm}^{0.90} / \mathrm{Pgm}^{1.00}: \mathrm{Pgm}^{1.00} / \mathrm{Pgm}^{1.00} \text {. }
\end{aligned}
$$

For $A d h$, the area within the 95 per cent contour included the origin (where $s=t=0$ ) for Redbanks and Craigmoor but $s$ was significantly positive for Rothbury and both $s$ and $t$ were significantly positive for Cygnet. In general the relative fitnesses of both $A d h^{F} / A d h^{S}$ heterozygotes and $A d h^{F} / A d h^{F}$ 
homozygotes were higher in base populations from more temperate latitudes, which implied that equilibrium $A d h^{F}$ frequencies would also be higher at more temperate latitudes.

The magnitudes of $s$ and $t$ estimates for $G p d h$ were generally greater than those for $A d h$ and only for Rothbury did the 95 per cent contour contain the origin. The relative fitnesses of $G p d h^{F} / G p d h^{S}$ and $G p d h^{F} / G p d h^{F}$, and consequently also the predicted equilibrium $G p d h^{+}$frequencies, were lower in base populations from more temperate latitudes.

For $P g m^{1 \cdot 20}-P g m^{1 \cdot 00}$ homogeneous results were obtained across base populations and the area within the 95 per cent contour excluded the origin, lying mainly within the sector of positive $s$ and $t$. This implied that the $P g m^{1 \cdot 00}$ homozygotes and heterozygotes generally had higher fitness than $P g m^{1 \cdot 20}$ homozygotes and that the equilibrium $P g m^{1.00}$ frequency was high, if not 100 per cent. Broadly similar results were obtained for $\mathrm{Pgm}^{0.90}$ $\mathrm{Pgm}^{1 \cdot 00}$, despite some slight differences between base populations. For this polymorphism the 95 per cent contour included the origin for all base populations except Craigmoor. The Craigmoor contour again indicated high relative fitnesses for $P g m^{1 \cdot 00}$ homozygotes and heterozygotes and high equilibrium $\mathrm{Pgm}^{1.00}$ frequencies. Contours for the other three populations were consistent with this but also did not exclude the hypothesis of neutrality.

\section{Short-term exposures to high temperatures}

Table 3 gives the average survival percentages of the three $A d h$ genotypes in each of the eight populations after the heat shock. Analysis of variance

Table 3 Means and standard errors over all cohorts of the survival percentages of the three $A d h$ genotypes in each of the eight populations tested.

\begin{tabular}{|c|c|c|c|}
\hline Population & $A d h^{+} / A d h^{F}$ & $A d h^{F} / A d h^{s}$ & $A d h^{s} / A d h^{s}$ \\
\hline \multicolumn{4}{|c|}{ Queensland $\left(27.5^{\circ} \mathrm{S}, 153.0^{\circ} \mathrm{E}\right)$} \\
\hline Redbanks & $21 \cdot 9 \pm 3 \cdot 4$ & $21 \cdot 3 \pm 2 \cdot 6$ & $22 \cdot 0 \pm 4 \cdot()$ \\
\hline Ipswich & $31 \cdot 4 \pm 2 \cdot 1$ & $30 \cdot 3+2 \cdot 4$ & $32 \cdot 8 \pm 8 \cdot 8$ \\
\hline average & $26 \cdot 7 \pm 2 \cdot 3$ & $25 \cdot 8 \pm 2 \cdot 1$ & $27 \cdot 9 \pm 2 \cdot 5$ \\
\hline \multicolumn{4}{|c|}{ New South Wales $\left(32 \cdot 8^{\circ} \mathrm{S}, 151 \cdot 3^{\circ} \mathrm{E}\right)$} \\
\hline Bellevue & $23 \cdot 0 \pm 2 \cdot 6$ & $27 \cdot 4 \pm 3 \cdot 8$ & $28 \cdot 1 \pm 4 \cdot 2$ \\
\hline Rothbury & $30 \cdot 3+4 \cdot 7$ & $32 \cdot 2 \pm 3 \cdot 8$ & $29 \cdot 4 \pm 3 \cdot 2$ \\
\hline Elliot & $33 \cdot 7 \pm 2 \cdot 5$ & $35 \cdot 6 \pm 2 \cdot 3$ & $36 \cdot 0 \pm 2 \cdot 6$ \\
\hline Lindeman & $32 \cdot 7 \pm 1 \cdot 9$ & $33 \cdot 1 \pm 1 \cdot 7$ & $32 \cdot 7 \pm 2 \cdot 0$ \\
\hline Tulloch & $25 \cdot 2 \pm 3 \cdot 9$ & $24 \cdot 6 \pm 3 \cdot 1$ & $31 \cdot 3 \pm 3 \cdot 6$ \\
\hline average & $29 \cdot 4 \pm 1 \cdot 3$ & $31 \cdot 2 \pm 1 \cdot 3$ & $31 \cdot 6 \pm 1 \cdot 5$ \\
\hline \multicolumn{4}{|c|}{ Tasmania $\left(43 \cdot 0^{\circ} \mathrm{S}, 147 \cdot 3^{\circ} \mathrm{E}\right)$} \\
\hline Cygnet & $14 \cdot 3 \pm 3 \cdot 2$ & $16 \cdot 3+1 \cdot 5$ & $19 \cdot 4 \pm 7 \cdot 9$ \\
\hline
\end{tabular}

on angular transforms of these data indicated no significant main effect of $A d h$ genotype $\left(F_{(2,179)}=\right.$ $0.59, P>0.05)$, nor any significant interaction between $A d h$ genotype and population $\left(F_{(14,179)}=\right.$ $0 \cdot 20, P>0.05)$. However, there was a significant main effect of population $\left(F_{(7,179)}=13 \cdot 06, \quad P<\right.$ $0.001)$, most ( 69 per cent) of which reflected the difference between the Cygnet population and the other seven. The lower survival of the Cygnet flies may relate to the more temperate latitude and cooler climate of the collection locality.

The average survival percentages $(\bar{x} \pm$ S.E.) after the heat shock for $G p d h$ genotypes from Cygnet were $7 \cdot 0 \pm 2 \cdot 3$ for $G p d h^{S} / G p d h^{s}, 17 \cdot 5 \pm 1 \cdot 7$ for $G p d h^{F} / G p d h^{S}$ and $29 \cdot 5 \pm 6.4$ for $G p d h^{F} / G p d h^{+}$. The differences between the three genotypes in (angularly transformed) survival percentages were clearly significant $\left(F_{(2,14)}=9 \cdot 45, P<\right.$ $0 \cdot 01)$. Thus, $G p d h^{F} / G p d h^{\digamma}$ homozygotes were about four times, and $G p d h^{+} / G p d h^{5}$ heterozygotes twice as likely to survive, as $G p d h^{s} / G p d h^{S}$ homozygotes.

\section{DISCUSSION}

Our first conclusion from the experiment exposing flies continuously to relatively mild temperatures is that the hypothesis of no selection is rejected by the data for each of the Adh, Gpdh and Pgm polymorphisms. At this level the results are consistent with previous multi-generation allele frequency perturbation experiments on these systems. However, the allele frequency changes reported in some of the previous experiments indicate convergence towards polymorphic equilibria, which has been taken as evidence of heterozygote advantage (Wilson et al., 1982 for Adh; PalabostCharles, 1982 for Gpdh and Carfagna et al., 1980 for $\mathrm{Pgm}$ ). While our Pgm data are generally consistent with this mode of selection, the estimates of selection coefficients for $A d h$ and $G p d h$ only clearly show heterozygote advantage in certain base populations (e.g., Cygnet and Redbanks respectively).

Some of these discrepancies between studies may reflect differences previously reported in the selective processes according to media type and the conditions of competition (van Delden et al., 1978; McKechnie et al., 1981; Charles-Palabost, 1982).

However, the present results support other evidence that such discrepancies may result from a more general phenomenon, involving systematic differences in selective processes among base 
populations according to the latitude of their collection. In an analogous multi-generation experiment to the present one, Oakeshott et al. (1984a) found that equilibrium $A d h^{F}$ frequencies predicted from maximum likelihood estimates of the selection coefficients were higher in base populations from more temperate latitudes. This was consistent with the direction of latitudinal clines in $A d h^{F}$ frequencies observed in natural populations (Oakeshott et al., 1982) and is again evident in the present experiment. Admittedly, the base populations for the present experiment were collected from essentially only three latitudes. However, the data also show a trend for lower equilibrium $G p d h^{F}$ frequencies in base populations collected from more temperate latitudes, which in turn is consistent with the direction of latitudinal clines in $G p d h^{F}$ frequency (Oakeshott et al., 1982; 1984b). And, on the other hand, estimates of equilibrium $\mathrm{Pgm}^{1.00}$ frequency in the present data are generally high for all base populations, which concurs with the high $\operatorname{Pgm}^{1.00}$ frequency found in most wild populations, irrespective of latitude (Oakeshott et al., 1981).

The fact that the selection on Adh and Gpdh, but not Pgm, differs systematically among base populations suggests that the dynamics of the first two systems are more sensitive to the effects of different genetic backgrounds than are those of Pgm. Certainly base populations from different latitudes are likely to differ systematically in their genetic backgrounds, given that latitudinal clines have been reported for several enzyme polymorphisms (Oakeshott et al., 1984b), chromosome inversions (Knibb et al., 1981) and morphological characters (David and Bocquet, 1975). However, it is uncertain whether the background genetic differences are important either because (irrespective of their chromosomal location) they affect the cellular environment of the enzymes under study, or because (irrespective of their biochemical effects) they are linked to, and in gametic disequilibrium with, the studied loci, so that their population dynamics affect those of the studied loci.

One closely linked element in the genetic background whose dynamics might interact with those of the Adh and Gpdh polymorphisms is the chromosome inversion $\operatorname{In}(2 L) t$. This inversion contains the Gpdh locus and is closely linked to Adh. Knibb (1983) have shown that $\operatorname{In}(2 L) t$ is in gametic disequilibrium with both loci and that its frequencies are clinally related to latitude. However, $\operatorname{In}(2 L) t$ only occurred in four of the 44 isofemale lines studied here (Redbanks 4, Redbanks 6, Craigmore 6 and Rothbury 8; Knibb, pers. comm.) and the dynamics of $A d h$ and $G p d h$ in these lines were not obviously different from those in lines lacking $\operatorname{In}(2 L) t$ (figs. 1 and 2). We conclude that $\operatorname{In}(2 L) t$ could have only made a minor contribution to the between line deviances for $A d h$ and $G p d h$ and that its contribution to the main effects of base population was probably negligible.

Nevertheless, it is inevitable that the dynamics of $A d h$ and $G p d h$ in this study were to some extent affected by other closely linked elements in the genetic background. Evidence is accumulating that contiguous genes may be as close as $5 \mathrm{~kb}$ of DNA, or less (e.g., Meyerowitz and Hogness, 1982), which may represent less than $0.01 \mathrm{cM}$ of recombination (Spradling and Rubin, 1981). Even without epistatic selection, gametic disequilibrium between sequences as close as this can readily be produced by chance events in small populations, for example during the founding of isofemale lines. And, once established, such disequilibrium will be relatively stable over time periods on the scale of the present experiment. The large deviance values recorded here between isofemale lines within base populations are therefore likely to reflect such background effects.

However, for gametic disequilibrium to account for the systematic differences between base populations as well, the disequilibrium would either have to be maintained by epistatic selection, or have been established at an early stage in the colonisation of Australia by D. melanogaster. Such a possibility cannot be dismissed, but without epistatic selection, its probability depends on very close linkage indeed.

The likelihood is therefore high that the differences among base populations in the selection on $A d h$ and $G p d h$ are due to the effects of background genes, not necessarily linked, on the cellular environments of the two enzymes. It remains obscure, however, why these effects should have been important for Adh and Gpdh, but not Pgm.

While the first experiment reported here yielded no evidence for selective effects of three relatively mild temperature environments, results from our second experiment did suggest effects of more severe temperature stresses, at least on the $G p d h$ polymorphism. In terms of adult survival after an hour at $38^{\circ} \mathrm{C}, G p d h^{F} / G p d h^{F}$ homozygotes were found to be more fit than $G p d h^{F} / G p d h^{S}$ heterozygotes, which in turn were more fit than $G p d h^{s} / G p d h^{s}$ homozygotes. These differences are consistent with the direction of latitudinal clines for $G p d h$ (since $G p d h^{F}$ increases in frequency towards warmer latitudes closer to the equator; 
Oakeshott et al., 1982;1984b) and with the direction of the difference in the thermal properties of the purified GPDH allozymes (Zera et al., 1983). The results are also consistent with the directions of differences in flying ability among the $G p d h$ genotypes at different temperatures (Barnes and Laurie-Ahlberg, 1985); this concurs with the well established role of GPDH in energy production in insect flight muscles (Zera et al., 1983 for a review).

For all these reasons we conclude that the selective effect of high temperature on the Gpdh polymorphism evident in our second experiment reflected direct effects on the locus, rather than on any linked loci in disequilibrium with $G p d h$. We suspect that similar effects were not detected in some earlier experiments (Milkman, 1977; Oakeshott, 1979; Schenfeld and McKechnie, 1979) partly because of differences in the size and power of the experiments and partly because of genuine differences in the selective effects of temperature shocks of different severity. Thus, in the present experiments, selective effects on $G p d h$ were not apparent even after 30 generations of continuous exposure to $29^{\circ} \mathrm{C}$ but were significant after only an hour's exposure of adults from a single generation to $38^{\circ} \mathrm{C}$ (notwithstanding that the first experiment encompassed all fitness components while the second only involved adult survival).

It is important, however, to ask whether selective processes like those observed on Gpdh in our second experiment are likely to occur in wild populations. The study populations should be representative of those in wild populations, since they were derived from very recent collections. Also relevant here are some of our unpublished results showing that adults of both sexes surviving an hour at $38^{\circ} \mathrm{C}$ remain fertile. Of course it must be conceded that this specific heat shock is unlikely to be encountered regularly by wild flies. However, the general consistency of the selection observed here with that reported in several (albeit not all) previous heat shock experiments indicates that the result is robust to some variation in the exposure conditions and may therefore have some relevance to natural populations.

Selective effects of different temperatures on the $A d h$ polymorphism were not detected in either of the present experiments, which is a similar result to that obtained in some previous work (Milkman, 1977; McKechnie et al., 1981; Vigue et al., 1982). Yet, other studies have produced evidence that the $A d h^{s}$ allele is more favoured at higher temperatures $\left(29^{\circ} \mathrm{C}\right.$ or above) than at lower ones (Johnson and Powell, 1974; van Delden et al., 1978; van Delden and Kamping, 1980; Sampsell and Sims, 1982). Discrepancies also emerge in comparisons among studies of seasonal variation in $A d h^{S}$ frequencies (Berger, 1971; Gionfriddo and Vigue, 1978; Franklin, 1981; Cavener and Clegg, 1981) and of the thermal properties of ADH allozymes (Zera et al., 1983 for a review); however, once again, where differences were found they generally suggested that the ADH-S allozyme and $A d h^{S}$ allele are better adapted to higher temperatures. There is thus suggestive but not conclusive evidence that selective effects of different temperatures underlie the negative relationship between $A d h^{S}$ frequency and distance from the equator.

One reason why the evidence for a direct selective effect of temperature is much less consistent for $A d h$ than for Gpdh may be related to the levels of electrophoretically cryptic variation at these loci. Whereas no such additional variation has been detected for Gpdh (Bewley, 1978), each of the two major electrophoretic variants of $A d h$ can be subdivided into at least three allelic classes on the basis of thermostability criteria (Sampsell, 1977). Although only one variant within $A d h^{S}$ is consistently found at frequencies above 1 per cent, two of the thermostability variants of $A d h^{F}$ commonly occur at polymorphic frequencies in natural populations (Wilks et al., 1980). The more common of these two ADH- $F$ variants is slightly less, but the rarer one considerably more, thermostable than ADH-S (Gibson et al., 1980).

It is interesting in this respect that no selective effects of temperature on the Pgm polymorphism were found in the present study. Admittedly there were no $\mathrm{Pgm}$ data for the $38^{\circ} \mathrm{C}$ heat shock experiment. However, the lack of effect of $29^{\circ} \mathrm{C}$ in the first experiment concurs with a similar result in another laboratory study (Oakeshott, 1979) and with the seasonal and latitudinal homogeneity of Pgm allele frequencies in wild populations (Cavener and Clegg, 1981; Franklin, 1981; Oakeshott et al., 1981). Thermostability variants occur at polymorphic frequencies within several of the electrophoretic variants of $P g m$, including the most common form, Pgm ${ }^{1 \cdot 00}$ (Trippa et al., 1976 ; 1978).

Thus the evidence for specific selective effects of temperature is convincing for $G p d h$, which apparently lacks cryptic variation, inconsistent for $A d h$, which has at least one polymorphic cryptic variant, and entirely lacking for Pgm, which has several such variants. Moreover, evidence is accumulating from the extensive application of thermostability and sequential gel electrophoretic analysis that many if not most enzyme polymorph. 
isms possess allelic variation additional to that detected by traditional electrophoresis (Keith, 1983). Now two points with fundamental implications must be made in this context. First, in theoretical terms, it has been shown that the range of selection coefficients which permit stable polymorphism is reduced rapidly as the number of alleles is increased (Crow and Kimura, 1970; Lewontin et al., 1978). Second, in practical terms, it is obvious that until studies such as the present one examine the full complement of alleles, their ability to resolve any selective mechanisms which do operate on the polymorphisms will be severely limited. This is particularly true for the investigation of the selective effects of temperature, since thermostability is one criterion which successfully detects electrophoretically cryptic variation.

Acknowledgements We thank Dr P. R. Anderson, Dr P. T. Barnes, Dr I. A. Boussy, Mr P. H. Cooke, Dr S. Easteal, Ms A. Y. Game, D. J. B. Gibson, Dr W. R. Knibb and Dr C. C. Laurie-Ahlberg for stimulating discussions and comments on the manuscript. Ms A. V. Wilks and Mr D. A. Willcocks provided excellent technical assistance.

\section{REFERENCES}

BARNES, P. T. AND LAURIE-AHLBERG, C. C. (1985). Genetic variation in flight metabolism of Drosophila: effects of Gpdh allozymes and temperature. Genetics (in press).

BERGER, E. M. (1971). A temporal survey of allelic variation in natural and laboratory populations of Drosophila melanogaster. Genetics, 67, 121-136.

BEWLEY, G. C. (1978). Heat stability studies of the $\alpha$ glycerophosphate dehydrogenase locus in populations of Drosophila melanogaster. Biochem. Genet., 16, 769-775.

CARFAGNA, M. FUCCI, L., GAUDIO, L., PONTECORVO, G. AND RUBINO, R. (1980). Adaptive value of PGM polymorphism in laboratory populations of Drosophila melanogaster. Genet. Res., 36, 265-276.

CAVENER, D. R. AND CLEGG, M. T. (1981). Temporal stability of allozyme frequencies in a natural population of Drosophila melanogaster. Genetics, 90, 629-644.

CHARLES-PALABOST, L. (1982). Influence du milieu sur le maintien du polymorphisme de l' $\alpha$-glycérphosphate déshydrogénase chez Drosophila melanogaster. Archiv. Zool. Exper. Générale, 122, 467-477.

CROW, J. F. AND KIMURA, M. (1970). An Introduction to Population Genetics Theory. Harper and Row, New York.

DAVID, J. AND BOCQUET, C. (1975). Evolution in a cosmopolitan species: genetic latitudinal clines in Drosophila melanogaster wild populations. Experientia, 31, 164-166.

FRANKLIN, I. R. (1981). An analysis of temporal variation at isozyme loci in Drosophila melanogaster. Gibson, J. B. and Oakeshott, J. G. (eds). In Genetic Studies of Drosophila Populations, pp. 217-236. Australian National University, Canberra.

GIBSON, J. B., CHAMBERS, G. K., WILKS, A. V. AND OAKESHOTT, J. G. (1980). An electrophoretically cryptic alcohol dehydrogenase variant in Drosophila melanogaster.
I. Activity ratios, thermostability, genetic localisation and comparison with two other thermostable variants. Aust. J. Biol. Sci., 33, 479-489.

GIONFRIDDO, M. A. AND VIGUE, C. L. (1978). Drosophila alcohol dehydrogenase frequencies and temperature. Genet. Res., 31, 97-101.

JOHNSON, F. M. AND POWELL, A. (1974). The alcohol dehydrogenases of Drosophila menalogaster: frequency changes associated with heat and cold shock. Proc. Nat. Acad. Sci. U.S.A., 71, 1783-1784.

KEITH, T. P. (1983). Frequency distribution of Esterase-5 alleles in two populations of Drosophila pseudoobscura. Genetics, $105,135-155$.

KNIBB, W. R. (1983). Chromosome inversion polymorphisms in Drosophila melanogaster. III. Gametic disequilibria and the contributions of inversion clines to the $\mathrm{ADH}$ and GPDH allozyme clines un Australia. Genetica, 61, 139-146.

KNIBB, W. R., OAKESHOTT, J. G. AND GIBSON, J. B. (1981). Chromosome inversion polymorphism in Drosophila melanogaster. I. Latitudinal clines and associations between inversions in Australasian populations. Genetica, 98, 833-847.

LEWONTIN, R. C., GINZBURG, L. R., TULJAPURKAR, S. D. (1978). Heterosis as an explanation for large amounts of genic polymorphism. Genetics, 88, 149-169.

MCKECHNIE, S. W., KOHANE, M. AND PHILLIPS, S. C. (1981). A search for interacting polymorphic enzyme loci in Drosophila melanogaster. In (Gibson, J. B. and Oakeshott, J. G. eds), Genetic Studies of Drosophila Populations, pp. 121-138. Australian National University, Canberra.

MEYEROWITZ, E. M. AND HOGNESS, D. S. (1982). Molecular organisation of a Drosophila puff site that responds to ecdysone. Cell, 28, 165-176.

MILKMAN, R. (1977). Temperature shock selection at three allozyme loci. Drosophila Inform. Serv., 52, 58.

Miller, S., PEARCY, R. W. AND Berger, E. (1975). Polymorphism at the $\alpha$-glycerophosphate dehydrogenase locus in D. melanogaster. I. Properties of adult allozymes. Biochem. Genet., 13, 175-188.

OAKESHOTT, J. G. (1979). Selection affecting enzyme polymorphisms in laboratory populations of Drosophila melanogaster. Oecologia, 43, 341-354.

OAKESHOTT, J. G., CHAMBERS, G. K., GIBSON, J. B. AND WILLCOCKS, D. A. (1981). Latitudinal relationships of esterase- 6 and phosphoglucomutase gene frequencies in Drosophila melanogaster. Heredity, 47, 385-396.

OAKESHOTT, J. G. AND GIBSON, J. B. (1981). Is there selection by environmental ethanol on the alcohol dehydrogenase locus in Drosophila melanogaster? In (Gibson, J. B. and Oakeshott, J. G. eds), Genetic Studies of Drosophila Populations, pp. 103-120. Australian National University, Canberra.

OAKESHOTT. J. G., GIBSON, J. B., ANDERSON, P. R., KNIBB, W. R., ANDERSON, D. G. AND CHAMBERS, G. K. (1982). Alcohol dehydrogenase and glycerol-3-phosphate dehydrogenase clines in Drosophila melanogaster on different continents. Evolution, 36, 86-96.

OAKESHOTT, J. G., GIBSON, J. B. AND WILSON, S. R. (1984a). Selective effects of the genetic background and ethanol on the alcohol dehydrogenase polymorphism in Drosophila melanogaster. Heredity, 53, 51-67.

OAKESHOTT, J. G., MCKECHNIE, S. W. AND CHAMBERS, G. K. $(1984 b)$. Population genetics of the metabolically related Adh, Gpdh and Tpi polymorphisms in Drosophila melanogaster. I. Geographic variation in Gpdh and Tpi allele frequencies in different continents. Genetica, 63, 21 29. 
OAKESHOTT, J. G., WILSON, S. R. AND GIBSON, J. B. (1983). An attempt to measure selection coefficients affecting the alcohol dehydrogenase polymorphism in Drosophila melanogaster populations maintained on ethanol media. Genetica, 61, 151-159.

PALABOST-CHARLES, L. (1982). Selection at the $\alpha$-Gpdh locus in experimental populations of Drosophila melanogaster. Biochem. Genet., 20, 461-474.

SAMPSELL, B. (1977). Isolation and genetic characterisation of alcohol dehydrogenase thermostability variants occurring in natural populations of Drosophila melanogaster. Biochem. Genet., 15, 971-988.

SAMPSELL, B. ANI) SIMS, S. (1982). Interaction of Adh genotype and heat stress on alcohol tolerance in Drosophila melanogaster. Nature, 296, 853-855.

SCHENFELD, E. AND MCKECHNIE, S. W. (1979). The $\alpha$ glycerophosphate dehydrogenase ( $\alpha$-gpdh) polymorphism in Drosophila melanogaster: adult survival under temperature stress. Theor. Appl. Genet., 54, 235-237.

SPRAILING, A. C. AND RUBIN, G. M. (1981). Drosophila genome organisation: conserved and dynamic aspects. Ann. Rev. Genet., 15, 219-264.

TRIPPA, G., CATAMO, G., LOMBARDOZZI, A. AND CICCHETTI, R. (1978). A simple approach for discovering common non-electrophoretic enzyme variability: a heat denaturation study of Drosophila melanogaster. Biochem. Genet., 16, 299-305.

TRIPPA, G., LOVERE, A. ANI) (:ATAMO, A. (1976). Thermostability studies for investigating non-electrophoretic polymorphic alleles in Drosophila melanogaster. Nature, 260,42-44.
VAN DELDEN, W., BOEREMA, A. C. AND KAMPING, A. (1978). The alcohol dehydrogenase polymorphism in populations of Drosophila melanogaster. I. Selection in different environments. Genetics, 90, 161-191.

VAN DELDIN, W. AND KAMPING, A. (1980). The alcohol dehydrogenase polymorphism in populations of Drosophila melanogaster. IV. Survival at high temperature. Genetica, $51,179-185$.

VIGUE, C. L.. WEISGRAM, P. A. AND ROSENTHAL, E. (1982). Selection at the alcohol dehydrogenase locus of Drosophila melanogaster: effects of ethanol and temperature. Biochem. Genet., 20, 681-688.

WILKS, A. V., GIBSON, J. B., OAKESHOTT, J. G. ANI CHAMBERS, G. K. (1980). An electrophoretically cryptic alcohol dehydrogenase variant in Drosophila melanogaster. II. Postelectrophoresis heat-treatment screening of natural pupulations. Aust. J. Biol. Sci., 33, 575-585.

WILSON, S. R. ANDOAKESHOTT, J. G. (1985). Statistical analysis of data from gene frequency perturbation experiments. Biometrical $J$. (in press).

WILSON, S. R., OAKESHOTT, J. G., GIBSON, J. B. AND ANDERSON, P. R. (1982). Measuring selection coefficients affecting the alcohol dehydrogenase polymorphism in Drosophila melanogaster. Genetics, 100, 113-126.

ZERA, A. J., KOEHN, R. K. AND HALL, J. (i. (1983). Allozymes and biochemical adaptation. Kerkut, G. A. and Gilbert, L. I. (eds). In Comprehensive Insect Physiology, Biochemistry and Pharmacology, Vol. 10. Pergamon Press, New York. 